\title{
Readability of Healthcare Literature for Gastroparesis and Evaluation of Medical Terminology in Reading Difficulty
}

\author{
Andrew Meillier ${ }^{\mathrm{a}, \mathrm{b}}$, Shyam Patel ${ }^{\mathrm{a}}$
}

\begin{abstract}
Background: Gastroparesis is a chronic condition that can be further enhanced with patient understanding. Patients' education resources on the Internet have become increasingly important in improving healthcare literacy. We evaluated the readability of online resources for gastroparesis and the influence by medical terminology.

Methods: Google searches were performed for "gastroparesis", "gastroparesis patient education material" and "gastroparesis patient information". Following, all medical terminology was determined if included on Taber's Medical Dictionary 22nd Edition. The medical terminology was replaced independently with "help" and "helping". Web resources were analyzed with the Readability Studio Professional Edition (Oleander Solutions, Vandalia, $\mathrm{OH}$ ) using 10 different readability scales.

Results: The average of the 26 patient education resources was $12.7 \pm$ 1.8 grade levels. The edited "help" group had $6.6 \pm 1.0$ and "helping" group had $10.4 \pm 2.1$ reading levels. In comparing the three groups, the "help" and "helping" groups had significantly lower readability levels ( $\mathrm{P}<0.001)$. The "help" group was significantly less than the "helping" group $(\mathrm{P}<0.001)$.

Conclusions: The web resources for gastroparesis were higher than the recommended reading level by the American Medical Association. Medical terminology was shown to be the cause for this elevated readability level with all, but four resources within the recommended grade levels following word replacement.
\end{abstract}

Keywords: Health literacy; Gastroparesis; Patient education as topic

\section{Introduction}

Patient education in long-term conditions has become a funda-

\footnotetext{
Manuscript accepted for publication January 26, 2017

aDepartment of Medicine, Temple University Health System, Philadelphia, PA, USA

${ }^{b}$ Corresponding Author: Andrew Meillier, 812 Parkinson Pavilion, Internal Medicine Department, Temple University Hospital, 3401 N Broad St, Philadelphia, PA 19140, USA. Email: Andrew.meillier@tuhs.temple.edu
}

doi: https://doi.org/10.14740/gr746w mental part of management. Communication has been instilled in improving understanding including the use of plain and non-medical language to ensure comprehension [1]. Healthcare literacy is considered as a constellation of skills to function in a healthcare environment [2]. On numerous prior studies, healthcare materials to help guide and educate the patient have been written at an exceedingly difficult level than what has been recommended [2]. Almost all patients prefer simple and easy to understand medical instructions [2]. The average adult reading level is eighth to ninth grade with one-fifth of the population able to read at the fifth grade reading level or below $[2,3]$.

Gastroparesis is a chronic condition of delayed gastric emptying without obstruction that is symptomatically characterized by nausea, vomiting, bloating and early satiety [4]. The condition requires consistent management with initial dietary modification that can help alleviate adverse effects on quality of life [5]. Prokinetic medications and gastric electrical stimulation are possible symptomatic treatments with surgical interventions considered for refractory cases with inability to fulfill nutritional requirements [5]. Patient understanding of the disease and symptomatic treatment with lifestyle changes can greatly improve quality of life. This can be further illustrated with patient educational materials that have increasing presence online.

The American Medical Association (AMA) has recommended writing patient education material at a sixth grade level or below. The writing construction should be written with short paragraphs and $1-2$ syllable words [1]. The US Department of Health and Human Services recommended sixth to seventh grade level with avoidance of medical terminology and complex words with simple sentences [6]. On numerous studies, online medical literature has been over the recommended reading level to appropriately inform the patient [7-9]. Limited studies have been performed to enact change as per the recommendations. The goal of this study was to determine the readability of patient education materials on web resources for gastroparesis using 10 different scales. Thereafter the medical terminology will be replaced to determine if any other changes are required to fulfill the recommended reading level for the average patient population.

\section{Methodology}

Google searches were performed for "gastroparesis", "gastroparesis patient education material", and "gastroparesis patient 
Table 1. Readability Grading Algorithms [9-18]

\begin{tabular}{|c|c|}
\hline Readability test & Formula \\
\hline $\begin{array}{l}\text { Automated readability } \\
\text { index (ARI) }[10,11]\end{array}$ & $\begin{array}{l}\text { Grade level }=0.50(\mathrm{~W} / \mathrm{S})+4.71(\mathrm{ST} / \mathrm{W})-21.43 \\
\mathrm{ST}=\text { letters, symbols and punctuation marks included (similar to total characters); } \mathrm{S}=\text { total sentence count; } \mathrm{W}= \\
\text { total word count }\end{array}$ \\
\hline $\begin{array}{l}\text { Bormuth grade placement } \\
\text { test [11] }\end{array}$ & $\begin{array}{l}\mathrm{R}=0.886593-0.083640(\mathrm{~L} / \mathrm{W})+0.161911(\mathrm{DLL} / \mathrm{W})^{3}-0.021401(\mathrm{~W} / \mathrm{S})+0.000577(\mathrm{~W} / \mathrm{S})^{2}-0.000005(\mathrm{~W} / \mathrm{S})^{3} \\
\mathrm{R}=\text { mean cloze score; } \mathrm{LET}=\text { total letters; } \mathrm{W}=\text { total words; } \mathrm{DLL}=\mathrm{Dale} \text { long list words; } \mathrm{S}=\text { total sentences }\end{array}$ \\
\hline $\begin{array}{l}\text { Coleman-Liau } \\
\text { index (CLI) [12] }\end{array}$ & $\begin{array}{l}\mathrm{CLI}=(0.0588 \times \mathrm{L})-(0.296 \times \mathrm{S})-15.8 \\
\mathrm{~L}=\text { average number of letters per } 100 \text { words; } \mathrm{S}=\text { average number of sentences per } 100 \text { words }\end{array}$ \\
\hline $\begin{array}{l}\text { Flesch-Kincaid } \\
\text { (FRE) [13] }\end{array}$ & $\begin{array}{l}\mathrm{FKGL}=(0.39 \times \mathrm{W} / \mathrm{SE})+(11.8 \times \mathrm{SY} / \mathrm{W})-15.59 \\
\mathrm{~W}=\text { total words; } \mathrm{SE}=\text { total sentences; } \mathrm{SY}=\text { total syllables }\end{array}$ \\
\hline FORCAST [11] & $\begin{array}{l}\text { Grade level }=20-(\mathrm{N} / 10) \\
\mathrm{N}=\text { number of single syllable words in a } 150 \text {-word sample }\end{array}$ \\
\hline Fry graph $[14]$ & $\begin{array}{l}\text { 1) Select three } 100 \text {-word passages from the beginning, middle and end. } \\
\text { 2) Sentences are counted in each passage and estimated to the nearest tenth and calculate the average. } \\
\text { 3) Count the total number of syllables in each } 100 \text {-word passage and calculate the average. } \\
\text { 4) Plot on the graph for determination of reading grade level. }\end{array}$ \\
\hline New Dale-Chall [16] & $\begin{array}{l}\mathrm{NDC}=0.1579 \mathrm{PC}+0.0496(\mathrm{~W} / \mathrm{S})+3.6365 \\
\mathrm{PC}=\text { percentage of complex words }(\text { words not on Dale-Chall word list }) ; \mathrm{W}=\text { total words; } \mathrm{S}=\text { total sentences }\end{array}$ \\
\hline $\begin{array}{l}\text { Raygor estimate graph } \\
{[17]}\end{array}$ & $\begin{array}{l}\text { 1) Select three } 100 \text {-word passages from the beginning, middle and end. } \\
\text { 2) Sentences are counted in each passage and estimated to the nearest tenth. } \\
\text { 3) Words with six or more letters are counted. } \\
\text { 4) The averages of the number of sentences and word length is plotted on the graph Raygor estimate graph. }\end{array}$ \\
\hline $\begin{array}{l}\text { SMOG readability } \\
\text { formula }(\mathrm{SMOG})[9,18]\end{array}$ & $\begin{array}{l}\mathrm{SMOG}=1.043 \times \sqrt{ }(30 \times(\mathrm{C} / \mathrm{S})+3.1291) \\
\mathrm{C}=\text { number of words with greater than two syllables; } \mathrm{S}=\text { total sentences }\end{array}$ \\
\hline
\end{tabular}

information". The first five search pages were viewed with exclusion criteria applied. Websites that were included were associated with professional organizations, medical search utilization resources, hospitals and universities. The resource was not required to originate in the United States. Internet resources that were excluded were public edited content, news related articles and blogs. The healthcare directed material was required to be in English. The articles were required to be comprised of sections for clinical symptoms, diagnosis, and treatment. Additional sections included if available were etiology, prognosis and dietary modifications. Other sections involving patient coping and questions for physicians were not included.

To eliminate changes in Google search optimization, all the searches were performed on the same day. Overall, 26 web resources fit the inclusion criteria. The resources included: American Academy of Family Physicians, American College of Gastroenterology, American Diabetes Association, Arizona Digestive Health, Barnes Jewish Hospital, Cleveland Clinic, Gastroparesis Patient Association for Cures and Treatments, Healthline, Indiana University Health, Intermountain Healthcare, International Foundation for Functional Gastrointestinal Disorders, John Hopkins Medicine, Mayo Clinic, Medical College of Wisconsin, MedicineNet, National Institute of Diabetes and Digestive and Kidney Diseases, National Organization of Rare Disorders, Temple University Hospital, The American Neurogastroenterology and Motility Society, The Children's
Hospital of Philadelphia, The University of Arizona Medical Center, University of California San Francisco Medical Center, University of Maryland Medical Center, University of Southern California, WebMD, and Western Sydney University. Once the web resources fit criteria, formatting was performed with removal of acknowledgements, references, author information, images and tables.

Readability Studio Professional Edition (Oleander Solutions, Vandalia, $\mathrm{OH}$ ) analyzed each article based on 10 readability scales: automated readability index (ARI), Bormuth grade placement test, Coleman-Liau, Flesch-Kincaid (FRE), FORCAST, Fry graph, Gunning Fog, new Dale-Chall, Raygor estimate graph, and SMOG (Table 1) [9-18]. The average reading grade level of the 10 scales was calculated. The formatted 26 web resources articles had all medical terminology replaced with "help" and "helping". Medical terminology criteria were determined using Taber's Medical Dictionary 22nd Edition (Unbound Medicine, Inc., Charlottesville, VA). Each article had every word searched using the online medical dictionary. The word that matched in the search was considered medical terminology if the root word and the definition corresponded to the use of the word in the article. If the root word matched, but was not in the same pre- or past tense, the word was considered medical terminology. The medical terminology was replaced by "help" and separately "helping". The three revised Internet resources were identified as unedited, help and helping groups. 


\section{Reading Level for Gastroparesis Online Resources}
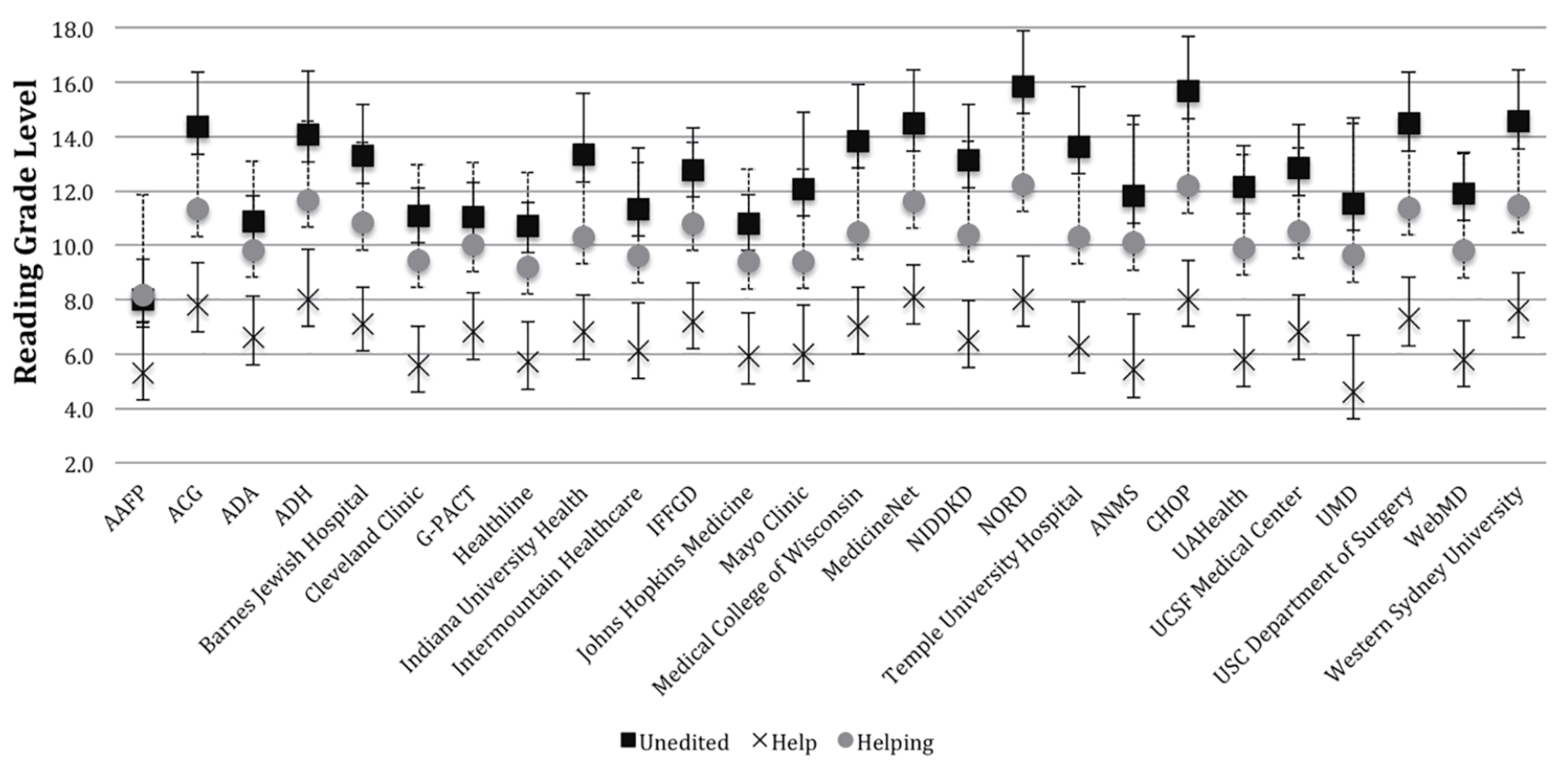

Figure 1. Reading level for gastroparesis online resources. The average grade level of the content from the 26 web resources with designated standard deviations. American Academy of Family Physicians (AAFP), American College of Gastroenterology (ACG), American Diabetes Association (ADA), Arizona Digestive Health (ADH), Barnes Jewish Hospital, Cleveland Clinic, Gastroparesis Patient Association for Cures and Treatments (G-PACT), Healthline, Indiana University Health, Intermountain Healthcare, International Foundation for Functional Gastrointestinal Disorders (IFFGD), John Hopkins Medicine, Mayo Clinic, Medical College of Wisconsin, MedicineNet, National Institute of Diabetes and Digestive and Kidney Diseases (NIDDKD), National Organization of Rare Disorders (NORD), Temple University Hospital, The American Neurogastroenterology and Motility Society, The Children's Hospital of Philadelphia (CHOP), The University of Arizona Medical Center (ANMS), University of California, San Francisco Medical Center (UCSF Medical Center), University of Maryland Medical Center (UMD), University of Southern California (USC), WebMD, and Western Sydney University.

The two new versions of the 26 articles were again analyzed using the 10 scales and the average reading grade level was determined.

The average grade levels of the 26 articles were transferred into SPSS version 22 (IBM Corporation, Somers, NY) with three separate categorizations of unedited, help and helping versions. Wilcoxon signed-rank test was performed comparing the original to the other versions of the articles separately. All P values less than 0.05 were considered statistically significant.

\section{Results}

A total of 26 gastroparesis patient education Internet resources were identified to match the required criteria. The unedited group had an average readability grade level of $12.7 \pm 1.78$ with a range of $5.5-18$ reading grade levels. The help group average was $6.6 \pm 1.0$ with a range from 2.4 to 7.8 reading grade levels and the helping group average was $10.4 \pm 2.1$ with a range of 3.0 - 17 reading grade levels. The National Organization of Rare Disorders average was 15.8 reading level with the largest difference among the edited groups. The help group had an 8.0 grade reading level and the helping group had a 12.2 reading level (Fig. 1). The new Dale-Chall readability scale showed the largest difference among the web resources' averages with a 4.8 grade level compared to the unedited group's 11.4 reading grade level.

The Academy of Family Physicians had the smallest difference between the unedited group 8.0 grade level and the help group with a 5.3 grade level (Fig. 1). The Academy of Family Physicians had the only increase in the readability level between the unedited group and helping group with reading grade levels of 8.0 and 8.2. This was largely due to the Raygor estimate graph with readability levels of grade $7-17$ between the unedited and helping groups. All other readability scales were equal or less except for FORCAST with 9.8 and 10.6 reading levels. Additionally, Academy of Family Physicians had the smallest difference between the help and helping groups. In comparing the help and helping groups, the largest improvement in readability was with the University of Maryland Medical Center with 9.6 - 4.6 reading grade levels.

In comparing the three groups, Wilcoxon signed-rank test was performed with the help group having a significantly lower readability grade average than the unedited and helping 
groups $(\mathrm{P}<0.001)$. All the Internet resources had been lowered with removal of the medical terminology with help. The helping group average was found to be significantly less than the unedited group $(\mathrm{P}<0.001)$.

\section{Discussion}

Patient education continues to play a fundamental role in quality of care especially for chronic conditions. Poorer health outcomes have been shown to occur with limited literacy and inability to understand health related information [1]. Low literacy was not related to physician visits, but more likely to report poor self health and number of hospitalizations [19]. Patients with limited health literacy have shown less knowledge of medical conditions, self-care and preventive measures [1]. Gastroparesis is a neuromuscular disorder that can require diet modification, antiemetic agents or parenteral nutrition and surgical therapy with refractory cases [5]. As a chronic condition, compliance with diet and medications can improve quality of life. The ability to conceive and understand the benefit can assist in patient behavior [20]. Patient education materials have been evaluated on prior studies showing consistent barriers with increased difficulty in required reading grade level to fully understand the text $[7,8,21]$.

Methods in improving understanding have dramatically changed in the last decade. In the current study, the first five Google search pages were used based on prior literature with majority of online users only viewing the first page [22]. The online resources were used with a recent national survey conducted by Pew Research Center finding that $59 \%$ of US adults look online for health information and eight out of 10 health inquires started on a search engine [23]. A study conducted in 2013 found that the first position on the Google search engine resulted in $33 \%$ of the traffic compared to $18 \%$ if located on the second position for the conducted search [22]. Additionally, sites listed on the first page of the search generated $92 \%$ of all traffic with a $95 \%$ drop of frequency on the second page [22].

In the current study, the 26 online resources averaged a reading grade level of $12.7 \pm 1.8$. This is above the AMA and US Department of Health and Human Services recommendations of patient education materials to be written at a sixth to seventh grade level $[1,6]$. None of the resources were at the recommended reading level. This is consistent with prior studies in evaluating patient education readability in other health topics $[9,24]$. The lowest reading level by the Academy of Family Physicians with an eighth grade reading level was likely influenced by being the shortest in total length among the online resources.

The 10 analysis methods in determining the readability of each online resource are based on one of four components: sentence length, word length, word syllables or complex words (Table 1). Complex words are not included on a list made by the creators of the algorithm. In evaluating medical literature, the word length, syllables and complex words can be considered likely influenced by the tendency of medical terminology to be longer with polysyllables. The elevated required reading level in patient education materials could be related to the in- clusion of medical terminology rather than the general format of the teaching material. This was previously evaluated with limited studies. One study used nursing students to remove what was considered medical terminology in five brochures and extended the passage by the number of words removed. This was analyzed by the SMOG and Fry readability scales with improvement, but not at the recommended reading levels [25]. A hand and wrist injuries study chose 10 articles and replaced medical terminology with the word "after". FleschKincaid and Dale-Chall readability scales were performed with two of the eight articles improving to the recommended reading levels [26].

In the present study, medical terminology inclusion was based on the Taber's Medical Dictionary. Every word included in the dictionary was removed and replaced with the word "help". All of the web resources significantly were reduced in readability level with the average of a 6.6 reading grade level. Additionally, every resource except 4 was within or below the recommended reading level for the general population. This illustrates that medical terminology plays a major part in the elevated readability levels. In analyzing the web resources with the replacement word "helping", the reading grade level was significantly reduced from 12.7 to 10.4 reading level average, but again, none of the web resources were within the recommended reading level. This shows that an improvement was made, but the second syllable and addition of three letters increased the reading difficulty significantly when compared to the help group.

Help and helping can be arguably similar in reading difficulty when compared outside of the readability scales. Replacement of the word help to helping significantly increased difficulty on the reading scales especially with Fry graph and Raygor estimate graph due to the algorithms including length and number of syllables (Table 1). This illustrates a limitation in this study with the word "helping" being similarly difficult in grading as the word "pleural" on certain scales yet significantly more difficult than "help". The commonality of the word is tested in the Bormuth grade placement test and new Dale-Chall readability tests. The new Dale-Chall word list included helping and the readability level average among the web resources was a 4.8 grade level compared to the unedited group's 11.4 reading grade level. This algorithm specifically only takes into account words not included on the simple word list and words per sentence. These algorithm restrictions do not fully comprise what can be considered all the components that create reading difficulty and require other reading scales to be taken into account.

The patient understanding remains critical to improving care and medical terminology should be individually considered when creating patient education materials. Although all complex terms in descriptions can be removed in effort of significant improvement in readability, practicality of deleting all medical terminology may not be helpful to the patient. In removing terminology, identifying the condition, medications and procedures would be more confusing to not use the appropriate names. Complexity and familiarity of words should have specific consideration when producing patient education information. This study has illustrated the level of difficulty in patient education materials has been largely based on medi- 
cal terminology and even in the setting of the studies limitations special considerations are needed. Overall, simplifying medical terminology would improve patient education materials and assist in patient healthcare literacy to promote better healthcare outcomes.

\section{Conclusion}

The 26 web resources for gastroparesis were higher than the recommended reading level of sixth to seventh grade. When the medical terminology was replaced with "help", all web resources except 4 were within the recommended reading level. Improvement in reducing medical terminology is critical in relaying patient education materials with specific consideration of word complexity to help improve better healthcare outcomes.

\section{Conflicts of Interest}

None.

\section{Grant/Financial Support}

None.

\section{Sources of Funding}

None.

\section{References}

1. Weiss B. Health literacy and patient safety: help patients understand: a manual for clinicians. 2nd ed. ed. Chicago, IL: American Association Foundation and American Medical Association; 2007.

2. Health literacy: report of the Council on Scientific Affairs. Ad Hoc Committee on Health Literacy for the Council on Scientific Affairs, American Medical Association. JAMA. 1999;281(6):552-557.

3. Brooks DA. Techniques for teaching ED patients with low literacy skills. J Emerg Nurs. 1998;24(6):601-603.

4. Pasricha PJ, Parkman HP. Gastroparesis: definitions and diagnosis. Gastroenterol Clin North Am. 2015;44(1):1-7.

5. Enweluzo C, Aziz F. Gastroparesis: a review of current and emerging treatment options. Clin Exp Gastroenterol. 2013;6:161-165.

6. How to write easy to read health materials. [Internet]. National Institutes of Health. [cited December 22, 2015]. Available from: http://www.nlm.nih.gov/medlineplus/etr. html.

7. Ibrahim AM, Vargas CR, Koolen PG, Chuang DJ, Lin SJ,
Lee BT. Readability of online patient resources for melanoma. Melanoma Res. 2016;26(1):58-65.

8. Meillier A, Patel S, Al-Osaimi AM. Readability of Healthcare Literature for Hepatitis B and C. Dig Dis Sci. 2015;60(12):3558-3562.

9. Hansberry DR, Ramchand T, Patel S, Kraus C, Jung J, Agarwal N, Gonzales SF, et al. Are we failing to communicate? Internet-based patient education materials and radiation safety. Eur J Radiol. 2014;83(9):1698-1702.

10. Smith EA, Senter RJ. Automated readability index. AMRL TR. 1967:1-14.

11. DuBay W. The Principles of Readability. 2004.

12. Coleman M LT. A computer readability formula designed for machine scoring. Journal of Applied Psychology. 1975;60(2):283-284.

13. Kincaid J, Fishburne RP, Rogers, RL, Chissom BS. Derivation of new readability formulas (automated readability index, fog count, and flesch reading ease formula) for Navy enlisted personnel. Chief of Naval Technical Training: Naval Air Station Memphis, 1975.

14. Fry E. A readability formula that saves time. Journal of Reading. 1968;11(7).

15. Gunning R. The Technique of Clear Writing. McGrawHill. 1952.

16. Chall J. Readability Revisited: The New Dale-Chall Readability Formula. Cambridge, MA: Brookline Books/ Lumen Editions; 1995.

17. Wolinksi J B, M. Using the Raygor Readability Estimate for Social Studies. The Social Studies. 1983;74(5):215219.

18. McLaughlin G. SMOG grading: A new readability formula. Journal of Reading. 1969;12(8).

19. Baker DW, Parker RM, Williams MV, Clark WS, Nurss J. The relationship of patient reading ability to self-reported health and use of health services. Am J Public Health. 1997;87(6):1027-1030.

20. Janz NK, Becker MH. The Health Belief Model: a decade later. Health Educ Q. 1984;11(1):1-47.

21. Shnaekel A, Hadden K, Barnes CL. Readability of Online Patient Educational Materials on Pain Management. J Surg Orthop Adv. 2015;24(4):242-245.

22. The Value of Google Result Positioning [Internet]. 2013 [cited January 15, 2016]. Available from: http://chitika. com/google-positioning-value.

23. Health Online 2013 [Internet]. 2013 [cited January 15, 2016.]. Available from: http://www.pewinternet. org/2013/01/15/health-online-2013.

24. Eberlin KR, Vargas CR, Chuang DJ, Lee BT. Patient education for carpal tunnel syndrome: analysis of readability. Hand (N Y). 2015;10(3):374-380.

25. Sand-Jecklin K. The impact of medical terminology on readability of patient education materials. J Community Health Nurs. 2007;24(2):119-129.

26. Wang SW, Capo JT, Orillaza N. Readability and comprehensibility of patient education material in hand-related web sites. J Hand Surg Am. 2009;34(7):1308-1315. 